\title{
New Design for a Versatile Field Microscope
}

\section{Yuval Goren}

Department of Archaeology \& Ancient Near Eastern Cultures, Tel Aviv University, Tel Aviv 69978, Israel

ygoren@post.tau.ac.il

\section{Introduction}

The present article presents a new concept for a light optical field microscope developed after two decades of attempts to find a portable, yet versatile and capable, instrument for extra-laboratory research. Emphasis was put on a portable microscope with polarizing capabilities, yet versatile enough to perform in other configurations. After testing almost every available model made during the last century, the Goren microscope, as it is called now, was developed and tested in various field conditions. The new design, fashioned as two prototypes, is expected to be inexpensive if commercially produced. Still, it can be readily modified to perform as a brightfield, dark-field, phase-contrast, or polarizing instrument. The historical background of field microscopes is briefly presented in context of this new invention.

\section{Historical Overview}

Early microscopes. The need for portable microscopes is nearly as old as the microscope itself. After the introduction of the microscope as a research tool during the seventeenth century by Antonie van Leeuwenhoek and Robert Hooke, its scientific role declined during the following century. Until the mid-nineteenth century the use of the microscope was a leisure pursuit for a bored aristocracy, both at home and in the open. The following decades, however, from the mid-nineteenth and into the twentieth century, can be regarded as the truly important age of all aspects of light microscopy including portables. This revolution began with the emergence of microbiology and optical mineralogy, the rise of exploration, and the demand of traveling scientists for rugged and capable instruments.

Early field microscopes. Field microscopy has always been a side branch of bench-top microscopy. However, with the increase of scientific exploration toward the middle of the nineteenth century, these instruments had to be adapted to the more demanding conditions of seafaring or long-range land transport. During the late nineteenth century, scientifically oriented portable microscopes with high-quality optics and durable design began to appear in response to users' demands. One of these instruments was a traveling microscope for naturalists designed by William Moginie, who worked with Charles Baker in London [1]. This design garnered almost instant popularity; it showed the importance of appropriate microtechnique in field work. This was later demonstrated by the work of Surgeon Major Sir Ronald Ross, who won in 1902 a Nobel Prize in medicine for his work on the life cycle of the malaria pathogen. Realizing the importance of a quality diagnostic portable instrument, Ross designed with Baker a successor to the original Baker-Moginie microscope for diagnosing malaria [2]. Like the earlier Moginie-style microscope, the apparatus was made portable by its smaller dimensions and folding tripod base, which became the archetypal concept for many field microscopes constructed up to World War II.
Another early twentieth-century concept was the pocket microscope. As opposed to the folding microscope, a pocket microscope is a miniaturization of the standard bench-top configuration of the compound microscope, preserving many of its features. The peak of success for pocket microscopes was between 1920 and the early 1930s. This was an era of exploration when expeditions into the interior of Africa, Asia, and South America were common along with field research in unorthodox conditions. German optical companies developed the new niche of compact, high-quality microscopes for the scientific community. However, these instruments were often extremely expensive, and their production ceased before World War II, never to be renewed significantly after the war [3].

Folded-optics design. The years after World War II were signified by one important event in the area of field microscopy: the introduction of the folded-optics design. This category refers to the exceptional construction of a handheld field microscope invented by John Norrie McArthur in the 1930s. This instrument was used by Dr. McArthur for malaria research in Borneo in 1937, and the final design was finally put on the market in 1957. Folded-optics microscopes of the McArthur concept have been produced by several makers. In the 1960s, Nikon produced the "Model $\mathrm{H}$," which was predominantly based on the principle of the McArthur microscope. Another McArthur-type model, the Swift FM-31, was introduced in 1981 [4]. Clones of the latter model are still produced today under several names, most likely in China [5].

Recent developments. From the 1980s and on, the concept of quality pocket or portable microscopy gradually subsided. Today, this niche is occupied either by nearly worthless toy microscopes or by compact versions of a biological bench-top apparatus, neither of which can satisfy the needs of a professional field microscopist. Although the family of the foldedoptics McArthur microscopes can be seen to some extent as a solution for the highly mobile scientist, it still presents many ergonomic and practical problems that make it inconvenient for prolonged scientific fieldwork. Current attempts to develop new field microscopes have yielded limited instruments that satisfy only a narrow aspect of microscopy, usually in the field of parasitology. As a result, the main microscope companies have abandoned the production of field microscopes, as if this type of instrument were no longer required by modern scientists. In some respect we were brought back to the early nineteenth century, when manufacturers rather than the scientists dictated the choice of microscopes.

Nevertheless, the needs of modern research and industry still call for the introduction of innovative, versatile, lowbudget, and effective field microscopes. Apart of the obvious need for a reasonable microscope for the analysis of tropical diseases in remote places, microscopes for extra-laboratory use can be applied to research in forensics, geology, soil research, art and archaeology, botany, off-lab microbiology, and quality 
control of materials in industry. Obviously, these disciplines require different configurations of field instruments variously employing bright field, dark field, phase contrast differential interference contrast, and polarizing capabilities-techniques that are rarely found in portable microscopes. Hence it would be desirable for field microscopes to be capable of modern imaging methods.

\section{A New Design}

The microscope design described here took into consideration the limitations of the earlier folding, pocket, and folded-optics microscopes. In order to achieve high versatility, multi-functionality, compact design, good ergonomics, and affordable production costs, an array of off-the-shelf parts was assembled. The key to portability is the substage design; several functions were combined into one integrated unit including the illumination element, the focusing mechanism, the substage optical system, and the stage. Into this combined unit various applications, in the form of sliders, are inserted to adjust the microscope within seconds to different requirements. Figure 1 presents the pocket version of the microscope with its accessories, made for proper fieldwork. Figure 2 shows the larger portable binocular version, made for more intricate work outside the laboratory. Both models are self-sustained, operated by batteries or, in the case of the heavier model, also by a USB power supply or $110 \mathrm{v} / 240 \mathrm{v}$ mains.

The configuration of the pocket version can be seen in the cross section of Figure 3. The substage illumination is by LED (1). Focusing is achieved by a helicoid (2), moving up and down (but not rotating) a cylinder that carries the stage (3). This cylinder has a slot beneath the stage for the application sliders (4) to be discussed later. The helicoid unit also houses the lower lens of the Abbe condenser (5), and the upper lens (6) is integrated within the stage. The upper optical system includes the usual ocular(s) and a turret with 3-4 objectives.

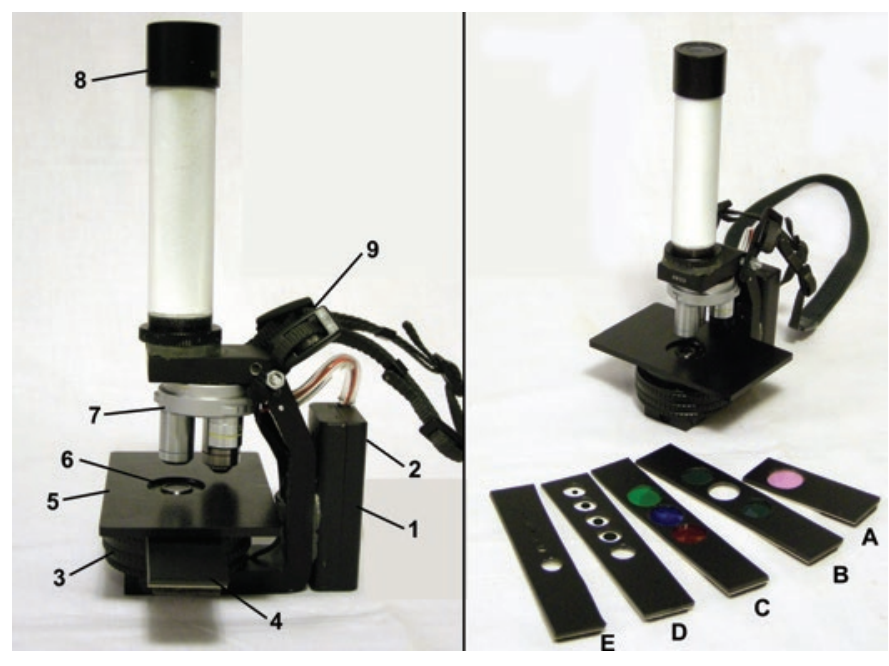

Figure 1: The pocket monocular version of the microscope with accessories. Left: (1) AA battery pack, (2) on/off switch to supply power, (3) focusing helicoid, (4) slot for application sliders, (5) broad stage for RMS and larger slides, (6) condenser, (7) three-objective turret, (8) ocular with an optional polarizer, (9) carrying strap. Right: application sliders including: (A) photographic filter; (B) a slider having two analyzer polarizers, one with an attached wavelength $\lambda$ ("tint") plate; (C) colored filters for bright field; (D) dark-field inserts; and (E) shutter slider.
In the larger instrument, Royal Microscopical Society (RMS) standard threads enable the use of any conventional objective lens. For the smaller version, the objective lens turret of a Swift FM-31 "clone" was used with three objectives $(4 \times$, NA0.08, working distance [w.d.] $16 \mathrm{~mm} ; 10 \times$, NA0.25, w.d. $3.6 \mathrm{~mm}$; $40 \times$, NA0.60, w.d. $0.6 \mathrm{~mm}$ ).

Figure 1 shows the pocket version with its parts (left) and some accessories (right). An AA battery pack (1) with an on/off switch (2) supplies the power. The helicoid (3) is for focusing, and

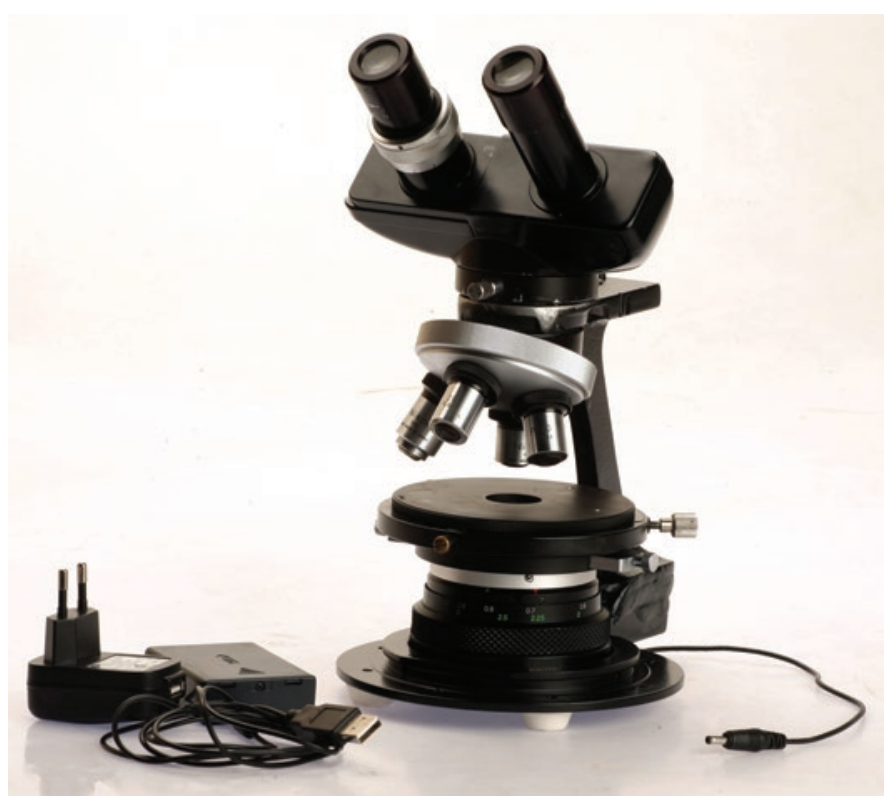

Figure 2: The portable binocular version of the microscope (photo: Pavel Shargo).

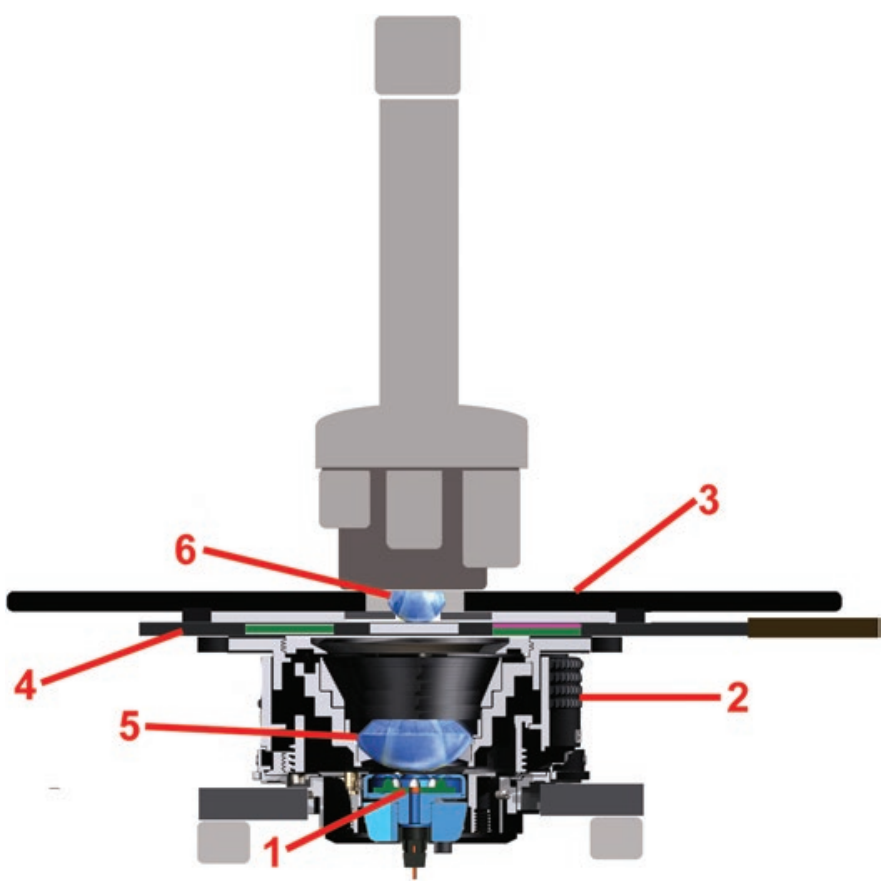

Figure 3: Cross section of the newly designed microscope: (1) LED substage illumination, (2) focusing helicoid for moving up and down (but not rotating) the stage, (3) stage, (4) substage slot for the application sliders, (5) lower lens, and (6) upper lens of the Abbe condenser. 


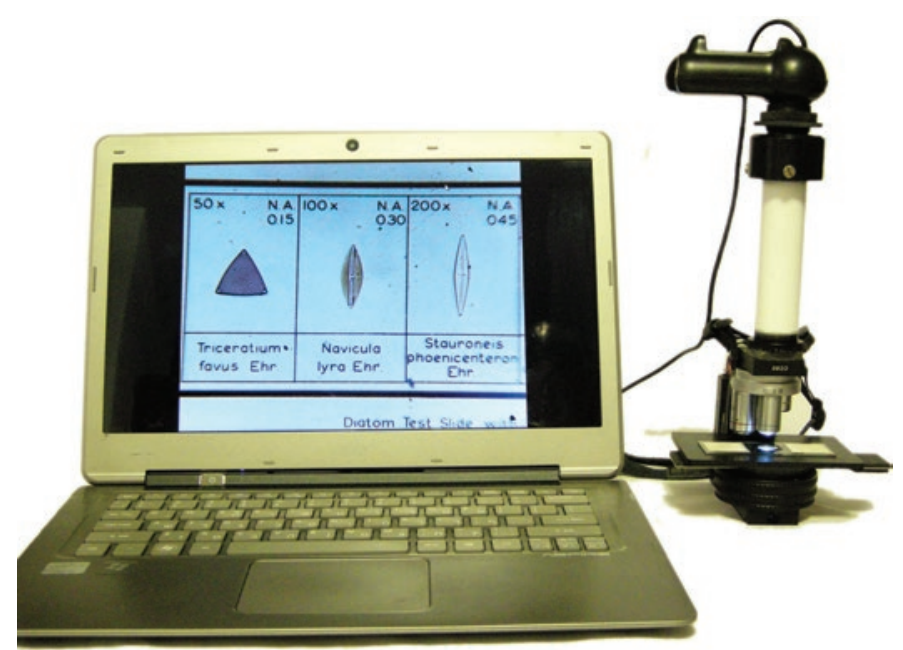

Figure 4: The microscope attached to a "poor man's improvisation" made with a 5 MP Logitech internet camera for image recording. The camera lens was removed and replaced by an attachment connecting it directly to the ocular. The image can be snapped by the laptop's camera software or grabbed by TWAIN into Photoshop or similar software.

the slot next to it is for the application sliders (4). A broad stage (5) enables the examination of RMS and larger slides, with the condenser (6) integrated in it. The tube contains a three-objective turret (7) and an ocular (8), which accepts an optional polarizer for petrographic applications, a crosshair, or a micrometer. Any RMS ocular can be used. A strap (9) enables hanging the microscope on the neck similarly to a camera or binoculars. Several application sliders are seen on the right including: photographic filter (A), a slider having two analyzer polarizers, one with an attached wavelength $\lambda$ ("tint") plate (B), color filters for bright field (C), dark-field inserts (D), and a shutter slider (E).

For field recording, any digital camera can be outfitted. In Figure 4 we see a "poor man's improvisation" made with a 5 MP Logitech internet camera, where the lens was removed and replaced by an attachment connecting it directly to the ocular. The image can be snapped by the laptop's camera software, or grabbed by TWAIN into Photoshop or similar software.

This microscope design offers a new concept that can be easily produced at low cost and still provide a versatile fieldwork kit for the serious scientist for many applications (Figure 5). Because it was made for our practical scientific work in microarchaeology (Figure 6), rather than designed by a commercial company, the Goren microscope can offer a reasonable solution for many aspects of modern microscopy in extra-laboratory conditions.

Two patent applications for the microscope are pending, submitted by Ramot Technology Transfer Company on behalf of Tel Aviv University and the author. A follow-up to this article will discuss the practical uses of this field microscope in various studies.

\section{Conclusion}

An historical survey examines the development of the field microscope in its several forms and shows that there is currently a lack of commercial interest in this type of instrument for scientists working outside the laboratory. This article describes a new design for a field microscope using such inexpensive modern components as an LED light source and digital camera

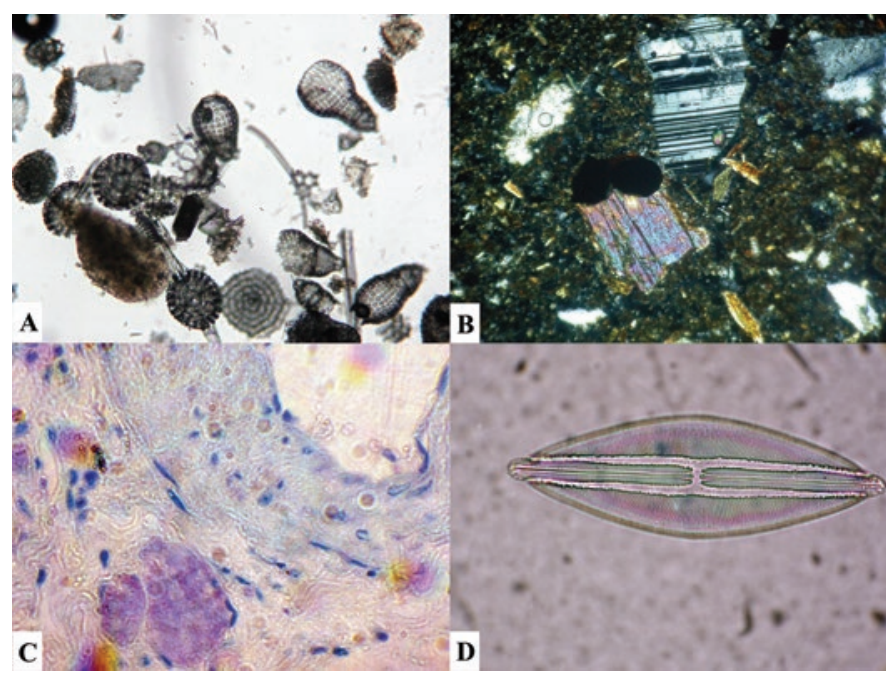

Figure 5: Photomicrographs taken under the Goren microscope of: (A) an array ("smear section") of radiolaria from a Middle Eocene deposit near Maresha, Israel (microscope magnification: 40×); (B) Field-impregnated and polished thin section of soil from Tsaghkasar, Armenia (100x, crossed polarizers); (C) Tuberculosis bacilli (600×, oil immersion); and (D) Diatom (Navicula Sp., 200×).

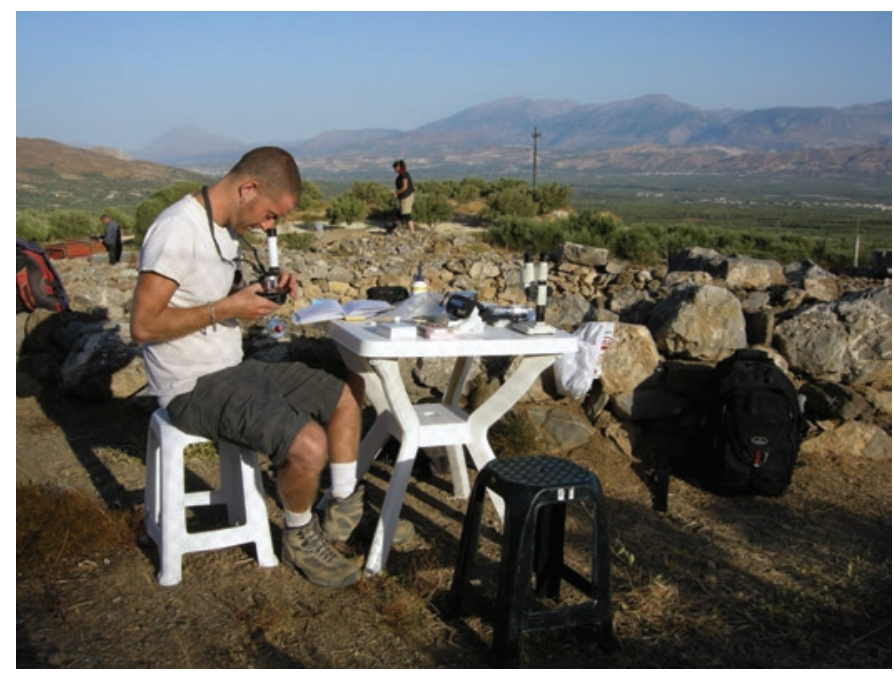

Figure 6: The microscope in use by micromorphologist Doron Bones (Tel Aviv University) for the analysis of ad-hoc sediment samples under crossed polarizers, in the course of the excavation of a Minoan cemetery at Koumasa (Crete) by the University of Heidelberg expedition (September 2012).

image acquisition. This instrument is capable of bright-field, dark-field, phase-contrast, and polarizing microscopy.

\section{References}

[1] RJ Kreindler and Y Goren, "Baker's Traveller's Microscopes," Micscape 187 (2011) http://microscopy-uk. org.uk/mag/indexmag.html.

[2] ME Gibson, T Roy Soc Trop Med H 92 (1998) 597-600.

[3] K Henkel, Mikrokosmos 89 (2000) 293-296.

[4] I Watt, Microscopy and Analysis 38 (1993) 27-29.

[5] RJ Kreindler and Y Goren, "Comparison of the Swift FM-31 Portable Field Microscope and an FM-31 Clone," Micscape 185 (2011) http://www.microscopy-uk.org.uk/ mag/artmar11/A_Comparison_of_FM-31_and_clone.pdf. 